\title{
Unsupervised cluster analysis of SARS-CoV-2 genomes reflects its geographic progression and identifies distinct genetic subgroups of SARS-CoV-2 virus
}

\author{
Georg Hahn ${ }^{*, 1}$, Sanghun Lee ${ }^{1,2}$, Scott T. Weiss ${ }^{3}$ and Christoph Lange ${ }^{1}$ \\ ${ }^{1}$ Department of Biostatistics, T.H. Chan School of Public Health, Harvard University, \\ Boston, MA 02115, USA \\ ${ }^{2}$ Department of Medical Consilience, Graduate School, Dankook University, South Korea \\ ${ }^{3}$ Channing Division of Network Medicine, Department of Medicine, Brigham and Women's \\ Hospital, and Harvard Medical School, Boston, MA 02115
}

\begin{abstract}
Over 10,000 viral genome sequences of the SARS-CoV-2 virus have been made readily available during the ongoing coronavirus pandemic since the initial genome sequence of the virus was released on the open access Virological website (http://virological.org/) early on January 11. We utilize the published data on the single stranded RNAs of 11,132 SARS-CoV-2 patients in the GISAID (Elbe and Buckland-Merrett, 2017, Shu and McCauley, 2017) database, which contains fully or partially sequenced SARS-CoV-2 samples from laboratories around the world. Among many important research questions which are currently being investigated, one aspect pertains to the genetic characterization/classification of the virus. We analyze data on the nucleotide sequencing of the virus and geographic information of a subset of 7,640 SARSCoV-2 patients without missing entries that are available in the GISAID database. Instead of modelling the mutation rate, applying phylogenetic tree approaches, etc., we here utilize a model-free clustering approach that compares the viruses at a genome-wide level. We apply principal component analysis to a similarity matrix that compares all pairs of these SARSCoV-2 nucleotide sequences at all loci simultaneously, using the Jaccard index (Jaccard, 1901; Tan et al., 2005, Prokopenko et al., 2016; Schlauch et al., 2017). Our analysis results of the SARS-CoV-2 genome data illustrates the geographic and chronological progression of the virus, starting from the first cases that were observed in China to the current wave of cases in Europe and North America. This is in line with a phylogenetic analysis which we use to contrast our
\end{abstract}

\footnotetext{
${ }^{*}$ Corresponding author: ghahn@hsph.harvard.edu
} 
results. We also observe that, based on their sequence data, the SARS-CoV-2 viruses cluster in distinct genetic subgroups. It is the subject of ongoing research to examine whether the genetic subgroup could be related to diseases outcome and its potential implications for vaccine development.

\section{Introduction}

Now, the outbreak of severe acute respiratory syndrome coronavirus 2 (SARS-CoV-2) has created a pandemic as it has spread swiftly from continent to continent, resulting in $>2,300,000$ infections, with approximately $5 \%$ mortality and a devastating effect on public health. Little is known about the rates of mutation in SARS-CoV-2, viral genetic variation is generated on the same timescale as virus transmission which allows us to track the spread of SARS-CoV-2. Phylogenetic and phylogeographic analysis conferred insights into distinct subtypes as the virus spreads through the global population and could be helpful in untangling complex virus transmission dynamics and understanding disease susceptibility (Mousavizadeh and Ghasemi, 2020).

One of the striking features of SARS-CoV-2 infection is the variable clinical features of the disease. In general, older persons seem to have greater severity and mortality (Zhou et al., 2020), as do males (Shi et al. 2020). However, some younger subjects have had fatal disease as well (Zhou et al. 2020). Organ involvement also varies with respiratory complications being most frequent but cardiac and renal complications occurring in some other patients (Zhou et al., 2020). This degree of clinical variation may be due to environmental factors such as confinement to a nursing home or chronic care facility (McMichael et al. 2020), or genetics of either the host or the virus. While studies of host genetics are underway, we have chosen here to focus on genetic variation of the virus.

By mid June 2020, the GISAID (Elbe and Buckland-Merrett, 2017; Shu and McCauley, 2017) database contained samples of the sequenced SARS-CoV-2 genome obtained from thousands of individuals infected with the human coronavirus disease. After routine cleaning and alignment of the data (see the Methods section for details), we were left with $n=7,640$ samples having a trimmed length of $p=28,784$ nucleotide sequences. In the principal component cluster analysis, we focused on the loci in the SARS-CoV-2 genome where differences to the SARS-CoV-2 reference 
sequence (in Hamming distance) are observed. The reference sequence is available on GISAID under the accession number EPI_SL_412026. The Hamming matrix indicates the mismatches to the reference sequence, and its row sums are the Hamming distance of each nucleotide sequence to the reference sequence.

As we are interested in the geographical/temporal change of the SARS-CoV-2 genome, we assessed the similarity between each pair of virus genomes with the Jaccard index (Jaccard, 1901, Tan et al., 2005). By computing the Jaccard index for each pair of samples based on all loci we constructed the Jaccard similarity matrix for the 7,640 virus genomes (Prokopenko et al., 2016, Schlauch et al. 2017). The Jaccard similarity matrix can efficiently be computed across a large set of genomes and is a powerful tool to detect genetic substructures/clusters in such sets Hahn et al. $2020 \mathrm{c}$ b).

The article is structured as follows. Section 2 describes the preparation of the dataset including cleaning and alignment, as well as the Jaccard similarity matrices we compute. Clustering results for the SARS-CoV-2 genome samples are presented in Section 3, where they are contrasted with a phylogenetic analysis. The article concludes with a discussion in Section 4.

\section{Methods}

\subsection{Data acquisition}

All analyses are based on nucleotide sequence data downloaded from GISAID (Elbe and BucklandMerrett, 2017, Shu and McCauley, 2017) on 14 June 2020. Only complete sequences (defined as having a length $\geq 29000$ ) were selected, resulting in 45,895 sequences.

\subsection{Data curation}

We removed sequences with reading errors in the fasta file (defined as letters other than A, C, G, T). Carrying out an exhaustive search for a reference sequence found a sequence of length 300 which we used as an anchor to align all samples. For this, we exhaustively enumerated all subsequences of the nucleotide sequences in our sample, and checked for each one of them if it is found at roughly the same position in all samples. The size of $300 \mathrm{bp}$ was chosen arbitrarily; however, this choice allowed us to accurately align all samples. 


\begin{abstract}
AATGTATACATTAAAAATGCAGACATTGTGGAAGAAGCTAAAAAGGTAAA ACCAACAGTGGTTGTTAATGCAGCCAATGTTTACCTTAAACATGGAGGAG GTGTTGCAGGAGCCTTAAATAAGGCTACTAACAATGCCATGCAAGTTGAA TCTGATGATTACATAGCTACTAATGGACCACTTAAAGTGGGTGGTAGTTG TGTTTTAAGCGGACACAATCTTGCTAAACACTGTCTTCATGTTGTCGGCC CAAATGTTAACAAAGGTGAAGACATTCAACTTCTTAAGAGTGCTTATGAA
\end{abstract}

We grouped samples together depending on the location tag in their fasta file using the following nine categories: (1) China (all provinces apart from Wuhan), (2) Wuhan, (3) Korea, (4) Europe (all countries apart from Italy), (5) Italy, (6) Africa, (7) Australia and New Zealand, (8) South(east) Asia, (9) North America (USA, Canada), (10) Central America (including Mexico), (11) South America, (12) Japan, and (13) Iran. Selecting only samples falling into those regions, having a complete time stamp (year, month, date), and having an exact match of the above reference sequence resulted in $n=7,640$ samples.

\title{
2.3 Trimming and comparison to reference sequence
}

After alignment of all $n$ samples with the anchor, we trimmed sequences to the left and right in order to establish a sequence window in which all sequences had reads. This window had a sequence length of $p=28,784$.

We denote with $X \in \mathbb{S}^{n \times p}$ the matrix of nucleotide sequences. Letting $r \in \mathbb{S}^{p}$ be the reference sequence published on GISAID (accession number EPI_ISL_412026), where $\mathbb{S}=\{A, C, G, T\}$, we compute a Hamming matrix $Y \in\{0,1\}^{n \times p}$ by defining $Y_{i j}=1$ if and only if $X_{i j} \neq r_{j}$, and $Y_{i j}=0$ otherwise. The matrix $Y$ indicates the mismatches to the reference sequence, and its row sums are the Hamming distance of each nucleotide sequence to the reference sequence.

\subsection{Measuring similarity}

We employ the locStra (Hahn et al., 2020c b) R-package to calculate the Jaccard similarity matrix of $Y$, denoted as $J:=\operatorname{Jac}(Y) \in \mathbb{R}^{n \times n}$. The Jaccard matrix measures the similarity between all pairs of the $n$ curated samples from GISAID. Computing the first two eigenvectors of $J$ (for plotting) completes the numerical part of the analysis. 


\section{Results}

Figure 1 displays the first two principal components of the Jaccard matrix for the analyzed SARSCoV-2 genomes, labelled with the country information obtained from GISAID. We observe four distinct branches/clusters for both European and North American samples (Fig.1, left). The top branch contains most cases from the US, the branch below is a mixture of US and European cases. The horizontal branch contains predominantly European cases, while the bottom branch contains a mixture of samples from Europe, China, and South(east) Asia. The predominantly European branch on the x-axis seems to be linked to the cluster at the origin, by genomes from Wuhan, Italy and Europe (Fig. 1, right).

Furthermore, we observe a dense cloud of points around the origin $(0,0)$, which contains indistinguishable data points for genomes from all countries of the data set. When looking at the zoomed-in region in Fig. 1 (right), we observe that starting from the origin $(0,0)$, which has the highest density of genomes from China and Wuhan, a spatial spread-out is seen in which genomes from geographically close locations (Korea and South/Southeast Asia) cluster in the neighborhood of the origin. This "original" cluster contains also a relatively large number of virus genomes from the US and Europe, which could suggest early transmissions to these geographic regions. Almost all of the data points for the genomes from Wuhan and China are part of this cluster around the origin $(0,0)$, reflecting where SARS-CoV-2 was first reported.

For a better understanding of the distinct clustering of the European and US samples into the four observed subbranches (Fig. 1, left), we examine the genomic locations where the four subgroups differ. In particular, we are interested in how SARS-CoV-2 samples belonging to those four branches compare to the SARS-CoV-2 reference sequence. For the reference genome subdivided into 50 consecutive bins, Fig. 2 shows the normalized numbers of mismatches when comparing the trimmed reference sequence to the four branches of European and American samples. The number of mismatches is normalized with respect to the bin size and the number of samples in each branch. We observe that, besides of the (outlier) mismatches at the beginning and at the end of the virus genome, the samples of the four distinct branches differ from the reference genome at the same four genomic regions, and by roughly similar frequencies. The only exception is the fourth branch at approximately position 25000, where the first two branches are similar, and the third and fourth 
branches are similar.

We also aim to contrast our results with the ones of a phylogenetic analysis, computed with the "TreeTool App" on the GISAID website (Freunde of GISAID, 2020) for GISAID samples falling into the same time range as the ones used in our analysis. The analysis with the TreeTool App is based on an alignment with MAFFT (Katoh, 2013), from which a phylogenetic tree is subsequently built using the tool "FastTree" (Price et al., 2010, Price, 2020). A comparison of our clustering in Figure 1 with the phylogenetic analysis in Figures 3 and 4 reveals notable similarities and differences.

We observe that, likewise to our approach, Figure 3 reveals four clusters (which emerge in roughly March 2020). The bottom one contains mostly a mix of Asian samples, similarly to the bottom cluster we observe. The second cluster (from the bottom) contains a mix of Asian and North American/ European samples, the third is mostly a mix of North American and European samples, and the top cluster contains mostly Asian and European samples. Here, the clusters in the center of the plot correspond to the middle cluster of mostly European samples that we observe in Figure 1. However, in contrast to Figure 3, our clustering analysis shows a stronger mixing of mostly European and North American samples in the top two clusters in Figure 1, whereas such a result is not observed for the phylogenetic tree.

While both approaches, principal components and phylogenetics, identify the same global clustering structures, the differences in the analysis results between the approaches are observed in the local clustering substructure. Given the limited data, the question which of the two approaches is preferable cannot be answered conclusively at this point. Amongst others, this decision will depend on the specifics of the application, the analysis question, and further verification of the results from either approach through external findings.

\section{Discussion}

Our model-free, global analysis of the SARS-CoV-2 sequences demonstrates that the genome of the virus varies by geographic region, with different viral sequences present in Asia vs. Europe and the US. Our analysis is complementary to model-based approaches, e.g. phylogenetic tree approaches, or estimation of mutation rates (Zhao et al., 2004). Our analysis results also suggest that there 
are four distinct genetic subgroups in Europe and the US. The viral sequences that were initially observed in Asia seem to be more homogeneous. The genetic sequences of viruses from Europe and the US are more diverse when compared to the sequences from countries in Asia. If new cases are observed, e.g. new cases in Beijing in June 2020 (Xiaohua, 2020; Liu and Nebehay, 2020), clustering approaches can help to understand the origin of the transmissions, as one can examine whether the genomes of the new cases belong to a previously observed cluster (Hahn et al., 2020a).

It is important to consider the methodological aspects of our analysis to describe the viral genetic diversity of SARS-CoV-2. We used a direct, unsupervised approach to compare the entire viral genomes, i.e. principle components analysis based on Jaccard similarity matrices. We did not include information about the geographic origin of the samples in the analysis nor did we attempt to directly model the evolutionary relationship of the different SARS-CoV-2 genomes, e.g. via phylogenetic analysis. Nevertheless, our analysis results reflect clearly the chronological spread of SARS-CoV-2 around the globe. Furthermore, the principal components that are obtained by our approach can be tested for dependency/association with other important variables, e.g. time. Moreover, while our approach currently does not allow for the incorporation of genomes with missing information, is our ongoing methodological work to allow for such an analysis. Both aforementioned directions are work in progress.

The advantages of our methodology compared to existing analyses are twofold: First, our unsupervised approach is capable of recovering geographic subgroups and geographic progression without any (spacial) information, and it takes all loci into account simultaneously. Second, it is particularly simple as it only involves the Hamming matrix and its Jaccard representation, both of which can be efficiently computed using binary operations only (Hahn et al., 2020b).

To be precise, the computational effort existing software such as "FastTree" (Price, 2020) is of the order of $O\left(n^{1.5} \log (n) p\right)$ (Price et al. 2010), where $n$ is the number of sequences and $p$ is the length of the alignment. In our approach, the computation of the Hamming matrix can be done in $O(n p)$, since it requires only the entry-wise comparison of the aligned reference genome to each trimmed nucleotide sequence. Afterwards, the computation of the Jaccard matrix on the Hamming matrix requires effort $O\left(s p n^{2}\right)$, see Hahn et al. (2020b), where $s$ is the sparsity of the input (Hamming) matrix. For the case of the SARS-CoV-2 analysis presented in this work, it turns out that after alignment, all SARS-CoV-2 sample sequences naturally share a lot of nucleotides, 
which is reflected in a proportion of non-zero entries of only 0.0067 for the GISAID data we analyze. The high sparsity effectively brings down the effort to an empirical effort of $O\left(n^{1.5} p\right)$, thus making our approach indeed more efficient than FastTree. For approximate computations, the calculation of the Jaccard matrix can be avoided altogether, leaving only the computation of the Hamming matrix in $O(n p)$, and an $O(n p)$ effort to calculate the first two eigenvectors using the technique of (Halko et al., 2011, Section 1.6).

Methodology to identify clusters in genomic data, using combination of discriminant analysis and prior dimensionality reduction via PCA, could be applied as future work (Jombart et al. 2010). However, the main goal of our submission is to show that a simple approach based on Hamming distance and Jaccard similarity matrices reveals a meaningful stratification of SARSCoV-2 genomes. Other approaches such as Lemey et al. (2020) focus on the incorporation of travel history data into the (phylogenetic) analysis, which is demonstrated for a small number of genomes. Such an approach can be more informative, however can be difficult to obtain travel history for thousands of samples. Here, we aimed instead to reflect a geographic progression and spatial clustering of SARS-CoV-2 based solely on genomic data.

Our analysis results have two potentially important implications. It is the subject of ongoing research to understand whether the four genetic subgroups of the SARS-CoV-2 viruses have different clinical implications in terms of disease progression and disease outcome.

One important research question in this context is how to link the sequenced viral genome information to clinical outcomes of Covid-19. In this context, we believe that the PCA clustering methodology could provide new insights as it enables the application of all the available methodologies that have been developed for genome-wide association studies (GWAS) in human genetics. For instance, one could conduct a genome-wide association study for Covid-19 in which the loci of the viral genomes are tested for association with (that is, regressed on) the clinical outcome variable of the host/patient. To guard such an analysis against confounding/substructure in the viral genome data, one can include the eigenvectors of the PCA analysis into the GWAS regression models. Initial applications of the GWAS methodology to sequenced SARS-CoV-2 genomes and their host's/patient's clinical data have identified loci in the SARS-CoV-2 genome that could be associated with mortality (Lange et al., 2020). Phylogenetic approaches do not necessarily offer this convenience. 
Furthermore, our results suggest that vaccine creation will have to take into account the genetic variability in viral sequence described here. As the virus has spread over time, its genetic diversity has increased and is likely to increase even further. To combat the virus successfully, it will be fundamental to understand the consequences of this development on the features of the virus.

\section{Acknowledgements}

The authors gratefully acknowledge the contributors, originating and submitting laboratories of

the sequences from GISAID's EpiCoV ${ }^{\mathrm{TM}}$ Database (Elbe and Buckland-Merrett, 2017; Shu and McCauley, 2017) on which this research is based. A detailed list of contributors is available in the Supplementary Information.

\section{Data Availability Statement}

Sequence data that support the findings of this study are deposited in the GISAID database with accession numbers in the range of EPI_ISL_402119 to EPI_ISL_467430 (https://www.gisaid.org/).

\section{Conflict of Interest}

The authors declare no conflict of interest.

\section{Funding}

The initial methodology work for this paper was funded by Cure Alzheimer's Fund; Funding for this research was provided through the National Human Genome Research Institute [R01HG008976]; and the National Heart, Lung, and Blood Institute [U01HL089856, U01HL089897, P01HL120839, P01HL132825, 2U01HG008685].

\section{References}

Elbe, S. and Buckland-Merrett, G. (2017). Data, disease and diplomacy: GISAID's innovative contribution to global health. Global Challenges, 1(33-46). 
Freunde of GISAID, e. (2020). Global Initiative on Sharing All Influenza Data - TreeTool App.

Hahn, G., Cho, M. H., Weiss, S. T., Silverman, E. K., and Lange, C. (2020a). Unsupervised cluster analysis of SARS-CoV-2 genomes indicates that recent (June 2020) cases in Beijing are from a genetic subgroup that consists of mostly European and South (east) Asian samples, of which the latter are the most recent. bioRxiv:2020.06.22.165936.

Hahn, G., Lutz, S., Hecker, J., Prokopenko, D., Cho, M., Silverman, E., Weiss, S., and Lange, C. (2020b). locstra: Fast analysis of regional/global stratification in whole genome sequencing (wgs) studies. Accepted for publication with Genetic Epidemiology. Preprint at bioRxiv:2020.03.06.981050.

Hahn, G., Lutz, S., and Lange, C. (2020c). locStra: Fast Implementation of (Local) Population Stratification Methods (v1.3). https://cran.r-project.org/web/packages/locStra/index. html.

Halko, N., Martinsson, P., and Tropp, J. (2011). Finding Structure with Randomness: Probabilistic Algorithms for Constructing Approximate Matrix Decompositions. SIAM Rev, 53(2):217-288.

Jaccard, P. (1901). Étude comparative de la distribution florale dans une portion des Alpes et des Jura. Bull. Soc. Vaud. Des. Sci. Nat., 37:547-579.

Jombart, T., Devillard, S., and Balloux, F. (2010). Discriminant analysis of principal components: a new method for the analysis of genetically structured populations. BMC Genetics, 11(94):1-15.

Katoh, K. (2013). Multiple alignment program for amino acid or nucleotide sequences.

Lange, C., Hahn, G., Wu, C. M., Lee, S., Hecker, J., Lutz, S. M., Haneuse, S., Qiao, D., Cho, M. H., Randolph, A., Laird, N. M., Weiss, S. T., Silverman, E. K., and Ribbeck, K. (2020). Mutations in SARS-CoV-2 spike protein and RNA polymerase complex are associated with COVID-19 mortality risk.

Lemey, P., Hong, S., Hill, V., Baele, G., Poletto, C., Colizza, V., O’Toole, A., McCrone, J. T., Andersen, K. G., Worobey, M., Nelson, M. I., Rambaut, A., and Suchard, M. A. (2020). Accommodating individual travel history, global mobility, and unsampled diversity in phylogeography: a sars-cov-2 case study. bioRxiv:2020.06.22.165464, pages 1-43. 
Liu, R. and Nebehay, S. (2020). China sees European virus strain in Beijing, WHO says more study needed. Reuters News (2020-06-18 9:26pm). https://www.reuters.com/article/us-healthcoronavirus-china-virus-data-idUSKBN23Q04L.

McMichael, T., Clark, S., Pogosjans, S., Kay, M., Lewis, J., Baer, A., Kawakami, V., Lukoff, M., Ferro, J., Brostrom-Smith, C., Riedo, F., Russell, D., Hiatt, B., Montgomery, P., Rao, A., Currie, D., Chow, E., Tobolowsky, F., Bardossy, A., Oakley, L., Jacobs, J., Schwartz, N., Stone, N., Reddy, S., Jernigan, J., Honein, M., Clark, T., and Duchin, J. (2020). COVID-19 in a LongTerm Care Facility - King County, Washington, February 27-March 9, 2020. MMWR Morb Mortal Wkly Rep, 69(12):339-342.

Mousavizadeh, L. and Ghasemi, S. (2020). Genotype and phenotype of COVID-19: Their roles in pathogenesis. Journal of Microbiology, Immunology and Infection, pages 1-5.

Price, M. N. (2020). Fasttree.

Price, M. N., Dehal, P. S., and Arkin, A. P. (2010). FastTree 2 - Approximately MaximumLikelihood Trees for Large Alignments. PLOS One, 5(3):e9490.

Prokopenko, D., Hecker, J., Silverman, E., Pagano, M., Nöthen, M., Dina, C., Lange, C., and Fier, H. (2016). Utilizing the Jaccard index to reveal population stratification in sequencing data: a simulation study and an application to the 1000 Genomes Project. Bioinformatics, $32(9): 1366-1372$.

Schlauch, D., Fier, H., and Lange, C. (2017). Identification of genetic outliers due to sub-structure and cryptic relationships. Bioinformatics, 33(13):1972-1979.

Shi, Y., Yu, X., Zhao, H., Wang, H., Zhao, R., and Sheng, J. (2020). Host susceptibility to severe COVID-19 and establishment of a host risk score: findings of 487 cases outside Wuhan. Crit Care, 24(1):108.

Shu, Y. and McCauley, J. (2017). GISAID: Global initiative on sharing all influenza data - from vision to reality. EuroSurveillance, 22(13).

Tan, P.-N., Steinbach, M., and Kumar, V. (2005). Introduction to Data Mining. Pearson; 1st Edition. 

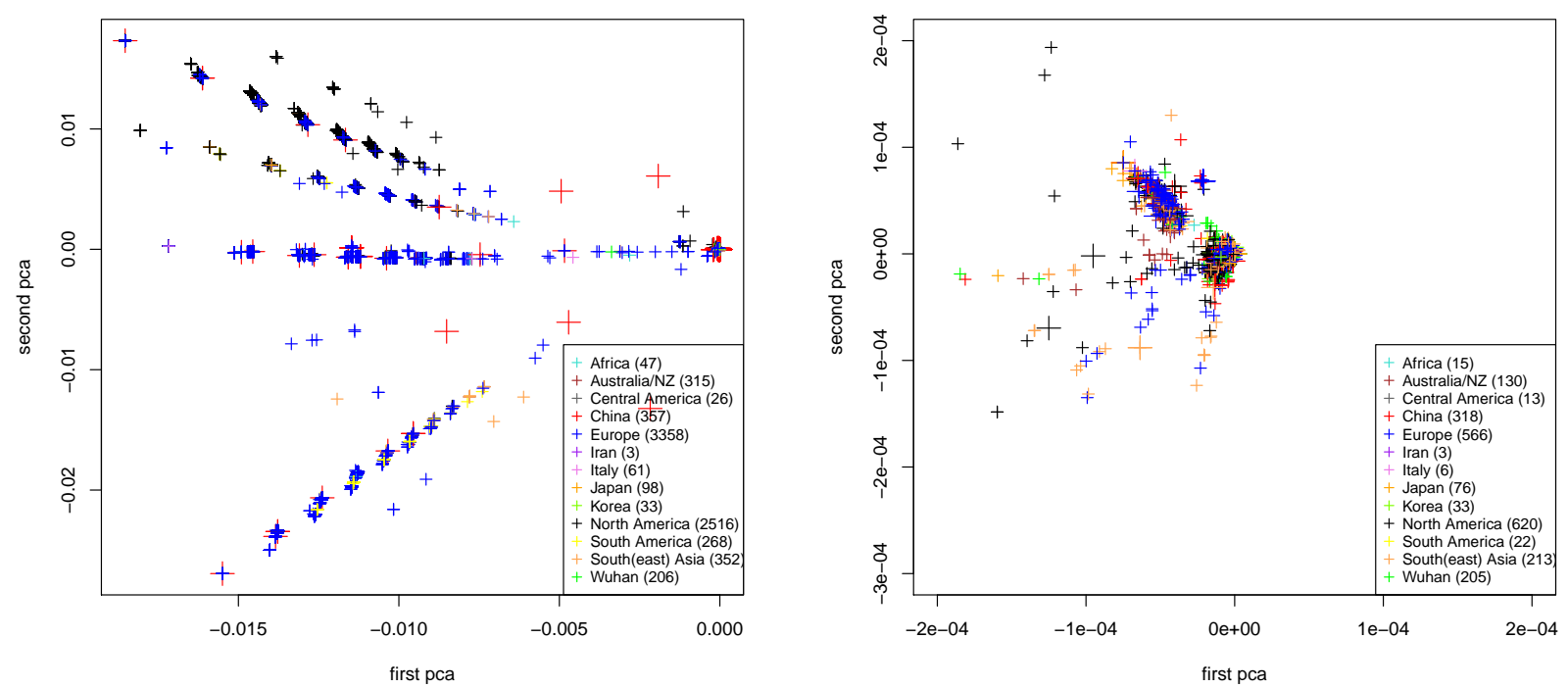

Figure 1: First two principal components of the Jaccard similarity matrix for the 7,640 SARSCoV-2 genomes by region/country. Entire dataset (left) and zoomed-in region around the origin $(0,0)$ (right). Numbers in brackets for each country denote the number of SARS-CoV-2 genomes which are visible in each plot.

Xiaohua, M. (2020). Experts decode the genome sequence released by CDC: Beijing new virus is imported. Yicai News (2020-06-19 6:14pm). https://www.yicai.com/news/100674046.html.

Zhao, Z., Li, H., Wu, X., Zhong, Y., Zhang, K., Zhang, Y.-P., Boerwinkle, E., and Fu, Y.-X. (2004). Moderate mutation rate in the SARS coronavirus genome and its implications. $B M C$ Evolutionary Biology, 4(21).

Zhou, F., Yu, T., Du, R., Fan, G., Liu, Y., Liu, Z., Xiang, J., Wang, Y., Song, B., Gu, X., Guan, L., Wei, Y., Li, H., Wu, X., Xu, J., Tu, S., Zhang, Y., Chen, H., and Cao, B. (2020). Clinical course and risk factors for mortality of adult inpatients with COVID-19 in Wuhan, China: a retrospective cohort study. Lancet, 395(10229):1054-1062. 


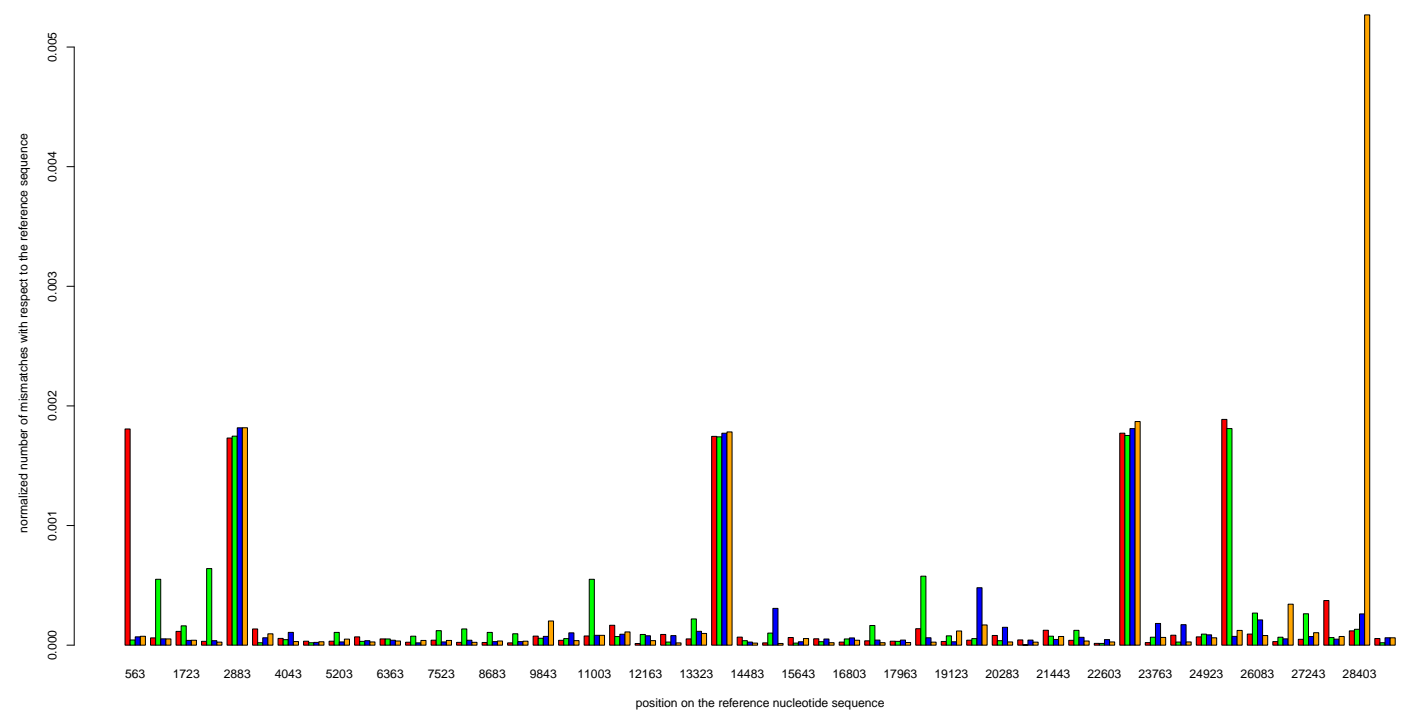

Figure 2: The x-axis shows the roughly 29, 000 nucleotides of the trimmed SARS-CoV-2 reference sequence in 50 bins. The y-axis shows per bin the normalized number of mismatches (with respect to the reference sequence) among the samples in the European and North American population, stratified into samples from the top branch (red), the second branch (green), the middle branch (blue), and the bottom branch (orange) visible in the left panel of Fig. 1. The normalization is done with respect to both the bin size and the number of samples in each branch.

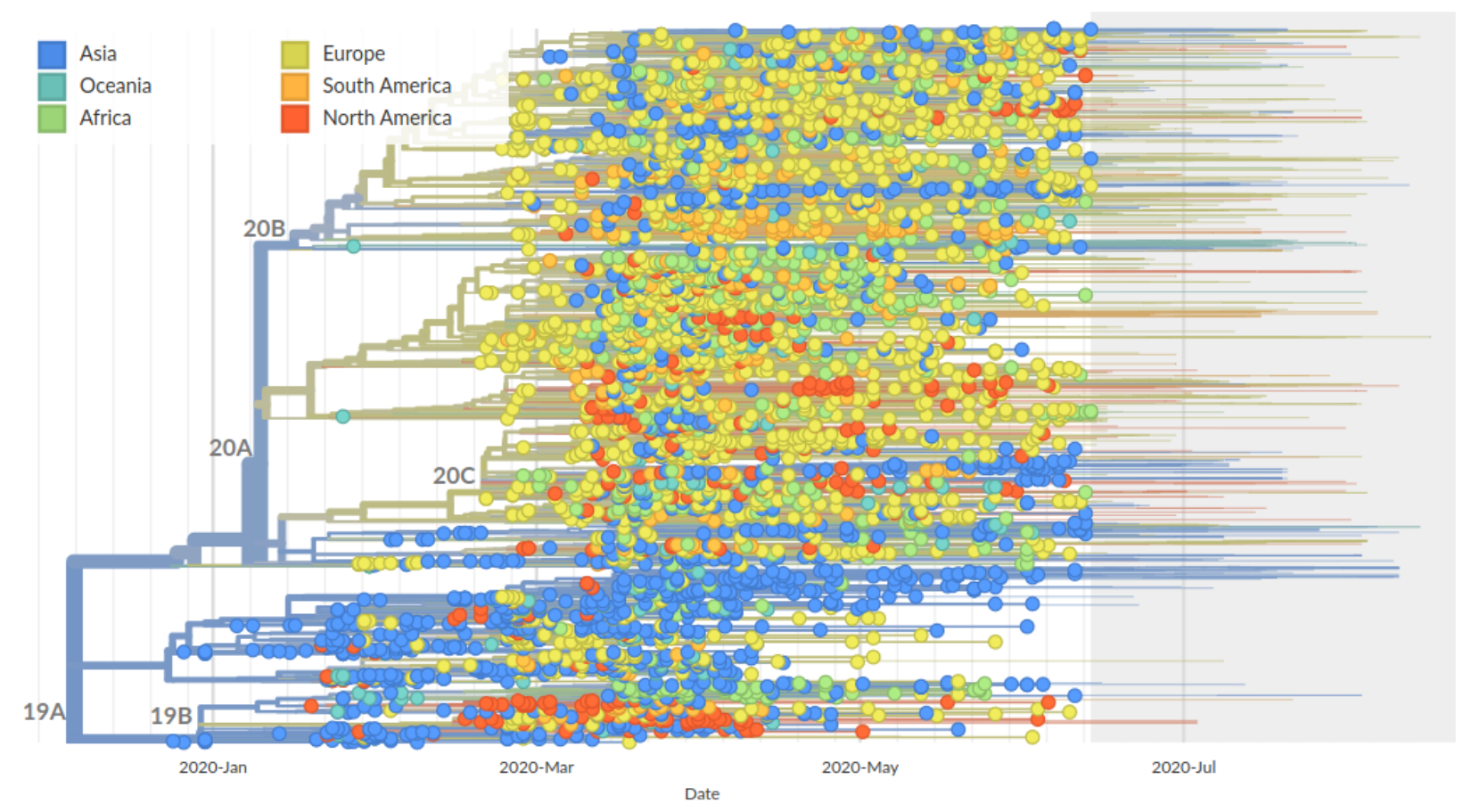

Figure 3: Phylogenic analysis as tree computed on https://www.gisaid.org/ epiflu-applications/influenza-phylogenetics/ using MAFFT for sequence alignment and FastTree for building the phylogenic tree. 
Asia

Oceania

Africa

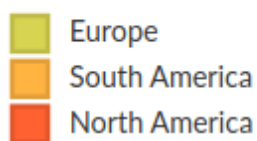

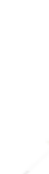
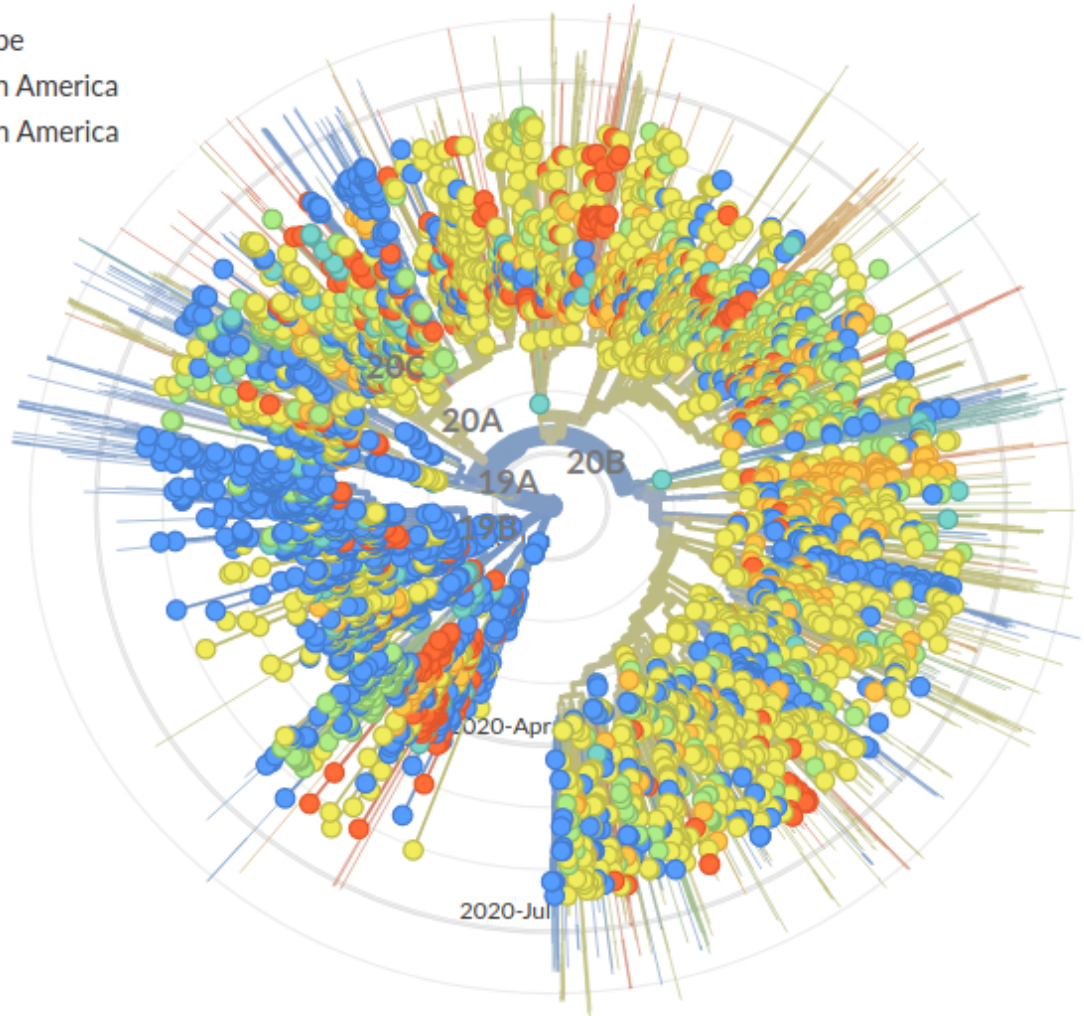

Figure 4: Phylogenic analysis as radial representation computed on https://www.gisaid.org/ epiflu-applications/influenza-phylogenetics/ using MAFFT for sequence alignment and FastTree for building the phylogenic tree. 